\title{
Using dispersion-induced group delay to solve the integer ambiguity problem: a theoretical analysis
}

\author{
D. Wei \\ weidong@mech.nagaokaut.ac.jp
}

\section{Xiao}

M. Aketagawa

\begin{abstract}
Department of Mechanical Engineering, Nagaoka University of Technology, Nagaoka City, Niigata 940-2188, Japan

School of Mechanical Engineering, Beijing Institute of Technology, Beijing 100081, People's Republic of China

Department of Mechanical Engineering, Nagaoka University of Technology, Nagaoka City, Niigata 940-2188, Japan
\end{abstract}

This paper describes a novel approach for solving the integer ambiguity problem when the adjacent pulse repetition interval length (APRIL) from a femtosecond optical frequency comb (FOFC) is used as a length scale. This approach is inspired by the two-color method, which indicates that there is a one-to-one relationship between the integer part of the APRIL and the group delay distance between the two different wavelengths. Accordingly, we numerically investigate the possibility of using dispersion-induced group delay to solve the integer ambiguity problem. The results of theoretical analyses and numerical investigations demonstrate the feasibility of the proposed method. Our results should contribute toward the further development of APRIL-based length measurement methods.

[DOI: http://dx.doi.org/10.2971/jeos.2015.15035]

Keywords: Dispersion, pulse repetition interval length, interferometry, integer ambiguity, metrology

\section{INTRODUCTION}

Currently, the standard SI unit of length, the meter, is defined in terms of the speed of light in a vacuum $(c)$. Under this definition, three methods have been recommended for realizing the meter, one of which used the wavelength of light. Owing to its frequency $(f)$ stability, the wavelength in a vacuum $(\lambda=c / f)$ of an iodine-stabilized helium-neon (He-Ne) laser is used to physically realize the meter. Recently, methods using the femtosecond optical frequency comb (FOFC) to realize the meter have been studied, along with its traceability. By stabilizing the carrier-envelope-offset frequency, $f_{C E O}$, and the repetition frequency, $f_{\text {rep }}$, the frequencies of an FOFC can be stabilized. Unlike a He-Ne laser, not only the wavelength but also the adjacent pulse repetition interval length (APRIL, $\left.\Lambda=c / f_{\text {rep }}\right)$ of the FOFC can be used to physically realize the meter [1]. An arbitrary and absolute length can be expressed in units of an APRIL as the sum of its integer part and its fractional part. Obviously, both parts are required for length information.

First, we consider the measurement of the fractional part. In a (balanced or unbalanced) Michelson interferometer, the pulse trains are reflected by the reference mirror and the object mirror, respectively. Only when they overlap with each other at the beam splitter is it possible to observe the peak of the envelope of the interference fringes. Thus, the position of the peak of the envelope indicates a point where the fractional part is zero [2]. For measuring an arbitrary fractional part, in an earlier report we proposed a multiple pulse train interferencebased interferometer [3]. When the interference fringes can be temporally [4]-[6] or spatially [7]-[10] separated, it is possible to determine the fractional part from the distance between the peaks of the envelopes of the interference fringes.

In this article, we consider the measurement of the integer part. In an earlier report, we reported that the integer ambiguity problem exists in APRIL-based distance determinations [11]. In a white-light based Michelson interferometer, because the white light only interferes with itself, by observing the peak position of the envelope of the interference fringes we can confirm that the reference mirror and the object mirror are located at the same distance from the beam splitter. In an FOFC-based (balanced or unbalanced) Michelson interferometer, because different pairs of pulse trains can interfere with each other, there is no one-to-one relationship between the position of the peak of the envelope and the relative positions between the reference mirror and the object mirror; this is not the case in a white-light interferometer. This phenomenon constitutes the integer ambiguity problem.

Therefore, in order to determine the integer part, two different scenarios have been proposed. One proceeds by shifting the repetition frequency $f_{\text {rep }}[2,12]$. There is a trade-off relationship between frequency variability and its stability; theoretically, we cannot expect to simultaneously achieve both longterm stability and short-term variability at the same time. To obtain short-term variability, the resonator of the light source must be able to be scanned at high speeds. On the other hand, in order to achieve long-term stability, the cavity of the laser must remain unchanged. These two facts conflict with each other. The other scenario involves the determination of the in- 
teger part by combining an interferometer with other optical methods, such as the time-of-flight method [13]. In this case, the disadvantages consist of the additional high-cost measurement devices that are required. As will be seen below, the proposed method requires only a pair of low-cost band-pass filters.

We attempt to address this task of determining the integer part based on the similarity between continuous wave (CW) white light and the FOFC source. First, we must explain what this similarity consists in. CW white light and FOFC lasers both have broad spectra, and both of them are classified as white light sources. The CW white light source has a continuous spectrum, whereas the FOFC laser, which is a stabilized-pulsed white light, has a discrete spectrum. For example, we assume that the center wavelength is $1560.0000 \mathrm{~nm}$, which corresponds to $192174652.5641 \mathrm{MHz}$. A $70.616 \mathrm{MHz}$ repetition frequency means that its adjacent frequency is 192174723.1801 MHz. Wavelength corresponding to the frequency is $1559.9994 \mathrm{~nm}$. Thus, there is a $\sim 6 \times 10^{-4} \mathrm{~nm}$ comb mode spacing between the two adjacent wavelengths. Currently, this mode spacing cannot be directly detected by optical measuring equipment, such as a photodetector and an optical spectrum analyzer. This fact means that both light sources can be used as a broad-band light source. Therefore, techniques based on CW white light can also be performed using an FOFC as a light source. It should be noted that comb-tooth resolving methods do exist and have been used for absolute distance measurement [14].

The purpose of this study is to make an estimation of the integer part. More specifically, we measure the group delay distance between the two color-band frequencies to achieve this purpose. Our supposition is that there is a one-to-one relationship between the integer part and the group delay distance. This idea was inspired by the two-color method [15][18], which is an accurate length-correction method that does not require the precise measurement of the environmental parameters. The two-color method can be understood as follows. When a displacement is caused by a change in the refractive index of air, this displacement can be corrected. The corrected length obtained by subtracting a value, which is a multiplication of an $A$ factor and a measured two-color length difference from a length obtained by one color. The length measured in air is proportional to the length in a vacuum, and the $A$ factor is only a function of the two colors used. This means that the measured two-color length difference is also proportional to the length in a vacuum. Accordingly, this is the primary reason why we suppose that there is a one-to-one relationship between the integer part and the group delay distance (namely, the two-color length difference). In the two-color method, the two-color length difference is used for correction. On the other hand, in the proposed method, the two-color length difference is used to obtain an estimation of the integer part. Since group delay is used in our method, our method only functions where dispersion exists.

This paper is organized as follows. Section 2 briefly reviews the length measurement based on the APRIL of an FOFC before describing the proposed method. Section 3 presents the numerical experiments. Finally, Section 4 summarizes the main conclusions.

\section{MATERIALS AND METHODS}

In this paper, we apply the fringe contour shift method $[19,20]$ to the FOFC-based method. First, we briefly introduce the fringe contour shift method. Pseudo-monochromatic (colorband) light sources were created by using a suitable bandpass filter with a white light source. As shown in Figure 1(a), the experiment $[19,20]$ was performed based on a Michelson interferometer in which the sample under test (e.g., a dielectric mirror, which introduced the frequency-dependent delay) was inserted in the object arm. By moving the reference mirror, one looks for a delay distance corresponding to the peak of the envelope of the interference signals appearing on the oscilloscope through a photodetector. By changing the central optical frequency of the band-pass filter, it is possible to continue to plot the delay distance, and one can obtain the group delay distance spectrum.

Based on the above-mentioned similarity, we apply the idea of the fringe contour shift method to a modified Michelson interferometer, which was proposed in Ref. [21]. As shown in Figure $1(b)$, the air in the unbalanced part of the interferometer introduces dispersion.

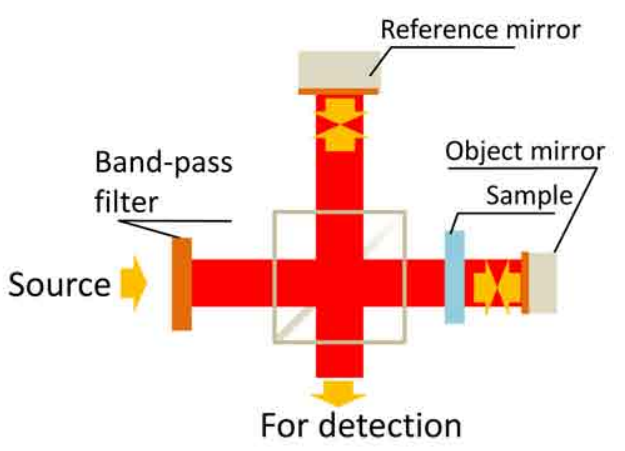

(a)

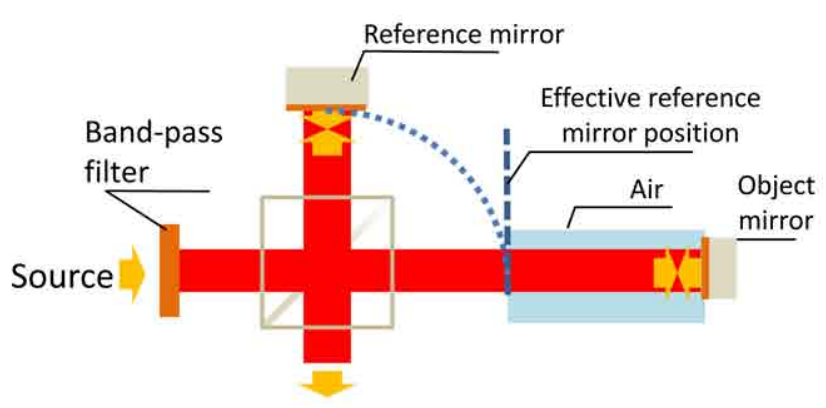

For detection

(b)

FIG. 1 Schematic of (a) the fringe contour shift method and (b) the proposed method. 


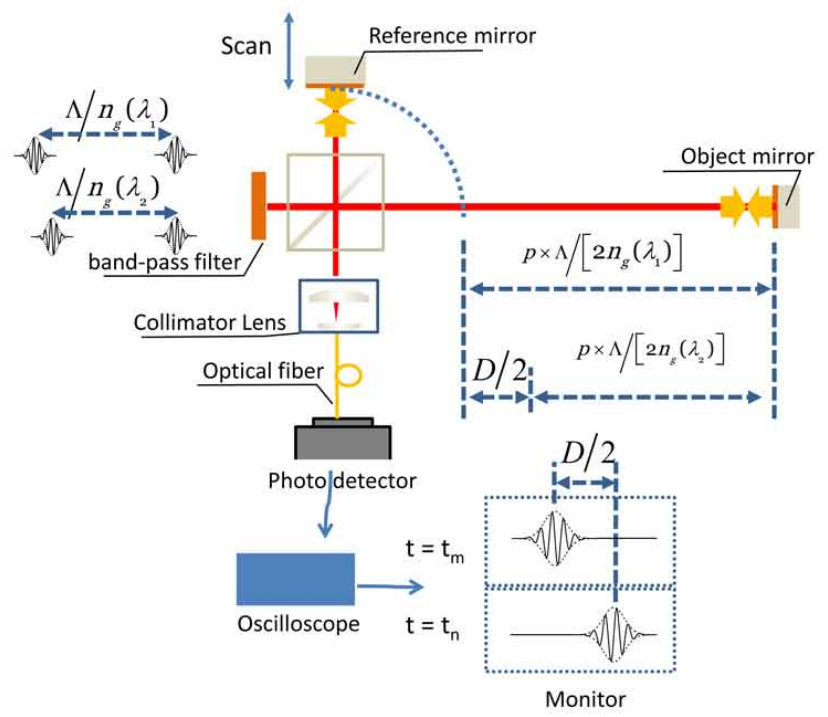

FIG. 2 Relative positional relationship between pulse trains when interference fringes can be observed. $D$ represents the dispersion-induced group delay distance between the two different frequencies.

For convenience, we start with a brief introduction of the FOFC; more details can be found in Ref. [22]. In the frequency domain, an FOFC laser can be modeled by a comb function, which is a combination of one million delta functions (namely, single frequency) arranged at equal intervals with the pulse repetition frequency $f_{\text {rep }}$. In the time domain, an FOFC emits a pulse train in which adjacent pulses are arranged at the time interval $T_{R}=1 / f_{\text {rep }}$, which corresponds to the APRIL, $\Lambda=c / f_{\text {rep }}$, in the spatial domain. In air, the APRIL is a function of wavelength.

The wavelength in air, $\lambda_{\text {air }}$, is a function of the wavelength in a vacuum, $\lambda_{v a c}$, and the phase refractive index of air, $n_{p}\left(\lambda_{v a c}\right)$, according to the equation $\lambda_{a i r}=\lambda_{v a c} / n_{p}\left(\lambda_{v a c}\right)$. In the same way, an absolute length $L$ measured in air using APRIL with a center wavelength of $\lambda_{i}$ can be expressed as:

$$
L_{i}=L / n_{g}\left(\lambda_{i}, T, P, H\right)=(p+q) \times \Lambda / n_{g}\left(\lambda_{i}, T, P, H\right) .
$$

Here, $p$ and $q$ are the integer and fractional part, respectively, obtained when $L$ is measured in units of $\Lambda$. T, $P$, and $H$ are the temperature, barometric pressure, and humidity, respectively. $n_{g}(\lambda)$ is the group refractive index, which can be calculated as [23]:

$$
\begin{aligned}
n_{g}\left(\lambda_{i}, T, P, H\right)= & n_{p}\left(\lambda_{i}, T, P, H\right) \\
& -\lambda_{i}\left[d\left(n_{p}(\lambda, T, P, H)\right) / d \lambda\right]_{\lambda_{i}} .
\end{aligned}
$$

Here, $n_{p}(\lambda)$ is the phase refractive index. In this report, values of $n_{p}(\lambda)$ are calculated using the Edln equation [24]. The equations for calculation can be found in Ref. [24].

Next, we consider how to obtain the dispersion-induced group delay distance between the two different frequencies. Let us consider the formation of interference fringes in the interferometer in Figure 2. Because $n_{g}\left(\lambda_{1}\right)$ and $n_{g}\left(\lambda_{2}\right)$ are different, we can assume that $n_{g}\left(\lambda_{1}\right)<n_{g}\left(\lambda_{2}\right)$; consequently, $L_{1}>L_{2}$. First, the band-pass filter is set to $\lambda_{1}$. Incoming pulses are split into two identical parts at the beam splitter. When the relative distance between the reference mirror and the object mirror is equal to $p \times \Lambda / n_{g}\left(\lambda_{1}\right)$ (round trip), the $i$ th pulse reflected from the object mirror and the $(i+p)$ th pulse reflected from the reference mirror will overlap and interfere with each other. As shown in Figure 2, the envelope of the interference fringes reaches its peak in this overlapped range. Next, the band-pass filter is set to $\lambda_{2}$. When the relative distance between the reference mirror and the object mirror is equal to $p \times \Lambda / n_{g}\left(\lambda_{2}\right)$, the $j$ th pulse reflected from the object mirror and the $(j+p)$ th pulse reflected from the reference mirror will overlap and interfere with each other. As shown in Figure 2, the envelope of the interference fringes reaches its peak in this overlapped range. Because the interference fringes are formed by different frequency slices, which are filtered out from the same FOFC source, the order of $p$ does not affect the formation of interference fringes. In order to observe the interference fringes, we need to scan the reference mirror. If the reference mirror is scanned far away from the beam splitter, as shown in Figure 2, we record the fringes of $\lambda_{1}$ first, then the fringes of $\lambda_{2}$ second. If we scan the reference mirror to the beam splitter, the order we observe the interference fringes in becomes reversed, which results in the observation of the fringes of $\lambda_{2}$ first, followed by the observation of the fringes of $\lambda_{1}$ second. An explanation of the formation of fringes and the detection of the envelope peak can be found in Refs. [3, 7].

The length difference between the two above-mentioned peaks of the two envelopes $D / 2$ is given by:

$$
\begin{aligned}
D & =p \times \Lambda / n_{g}\left(\lambda_{1}\right)-p \times \Lambda / n_{g}\left(\lambda_{2}\right)=p \times \Lambda \times B \\
B & =\left[n_{g}\left(\lambda_{2}\right)-n_{g}\left(\lambda_{1}\right)\right] /\left(n_{g}\left(\lambda_{1}\right) \times n_{g}\left(\lambda_{2}\right)\right)
\end{aligned}
$$

Obviously, the length difference between the two different frequencies is directly proportional to the integer part. That is because we assume that $n_{g}\left(\lambda_{1}\right)<n_{g}\left(\lambda_{2}\right)$, $p \times \Lambda / n_{g}\left(\lambda_{1}\right)>p \times \Lambda / n_{g}\left(\lambda_{2}\right)$, and therefore, $D>0$. We assume that reference mirror is scanned in the direction away from the beam splitter. The interference fringes of $\lambda_{1}$ are closer to the start scanning position than those of $\lambda_{2}$. If we assume that $n_{g}\left(\lambda_{1}\right)>n_{g}\left(\lambda_{2}\right), p \times \Lambda / n_{g}\left(\lambda_{1}\right)>p \times \Lambda / n_{g}\left(\lambda_{2}\right)$, then we have $D>0$. In this case, the interference fringes of $\lambda_{2}$ are closer to the start scanning position than those of the $\lambda_{1}$.

In the simulation described in the next section, we confirm the distinguishability between $(p+1) \times \Lambda \times B, p \times \Lambda \times B$, and $(p-1) \times \Lambda \times B$ under different situations.

A perfect beam splitter does not exist. Therefore, the pulses are never identical. Accordingly, imperfections of the beam splitter affect the signal-to-noise ratio. Minor vibrations in a scanning Michelson interferometer may affect the accuracy of the measurement. For simplicity, we explain the principle by using interference in the time domain. Interference systems in the frequency domain are also possible. However, it should be stressed that these sources of error (vibrations in an interferometer, scanning error of the reference mirror, etc.) are not related to the proposed method itself. An uncertainty budget will be provided in a separated paper. 


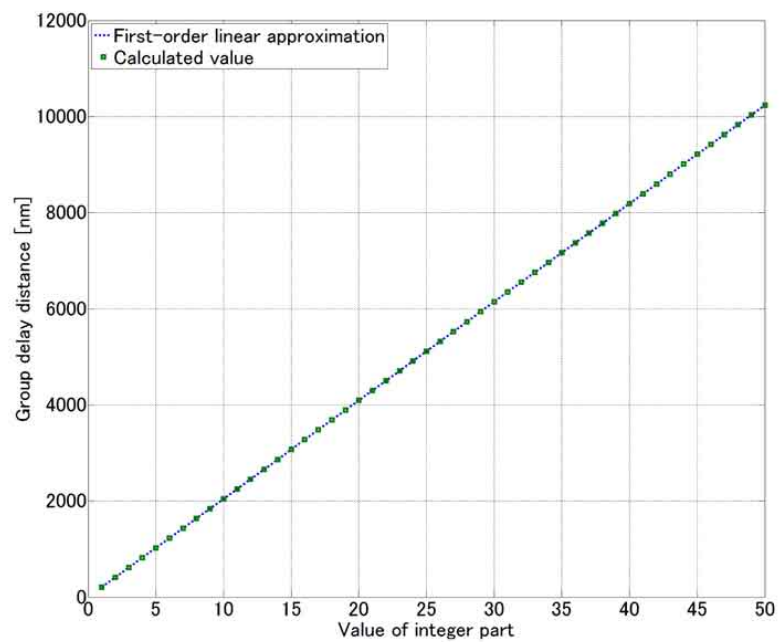

FIG. 3 Calculated group delay distance under standard environmental conditions.

\section{NUMERICAL SIMULATIONS}

We used the following parameters for the simulations. The APRIL was set to $4.245 \mathrm{~m}$ for an FOFC with a $70.616 \mathrm{MHz}$ repetition frequency. The two wavelengths were $1550 \mathrm{~nm}$ and $1570 \mathrm{~nm}$. These two wavelengths were selected based on the commercial availability of the band pass filters. The variable range of these two wavelengths was limited by the bandwidth of the FOFC source. Generally speaking, the difference between two separated wavelengths increases; the distance between the two peaks of the interference fringes also increases. This means that the required resolution of the measurement decreases. On the other hand, the travel range of the scanning mechanism increases. We used the same procedure [25] to calculate the group refractive index for these two wavelengths. Based on Eq. (3), we obtain the group delay distance between the two colors for the different values of $p$. We note that, owing to the nonlinearity of dispersion, the theoretical result can be slightly affected by the bandwidth and shape of each wavelength [26].

The first calculation was performed under standard environmental conditions (a temperature of $20^{\circ} \mathrm{C}$, a pressure of $101.325 \mathrm{kPa}$, and $50 \%$ humidity). The calculated value of the group delay distance (square points) and the first-order linear approximation curve (dotted line, based on Eq. (3)) are shown in Figure 3. The group delay distance varies proportionally with the value of the integer part $p$. This result is consistent with Eq. (3). In short, the higher the value of $p$ becomes, the wider the group delay distance becomes. We can conclude that under a well-controlled, well-measured environment, by measuring the group delay distance, we can find the value of p.

In cases where the distances are quite long, or in open-air conditions for which the environmental parameters must be measured with a certain amount of error, the variation of $n_{g}(\lambda)$ along the path may cause error. As shown in Figure 4, the calculated group delay distance is plotted against the temperature change (a temperature of $15-25^{\circ} \mathrm{C}$, a pressure of $101.325 \mathrm{kPa}$, and $50 \%$ humidity) for different values of $p$. For
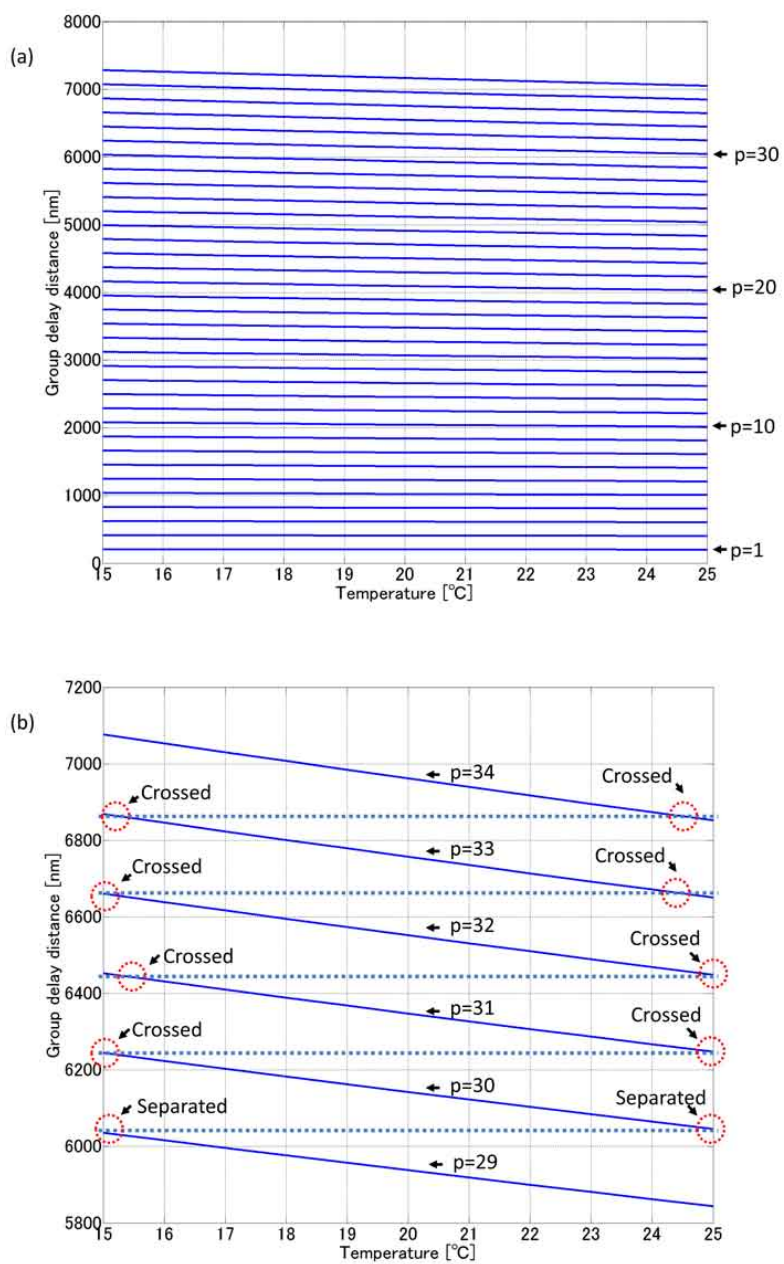

FIG. 4 (a) Calculated group delay distance versus temperature. (b) Enlarged part of (a) when the value of $P$ ranges from 30 to 34 (dotted lines are guides for the eye).

values of $p$ that are less than 30, the ranges of the calculated group delay distances do not overlap. When the value of $p$ is set at 31, the calculated group delay distance becomes 6453 $\mathrm{nm}$ at $15^{\circ} \mathrm{C}$. When the value of $p$ is set at 32 , the calculated group delay distance becomes $6449 \mathrm{~nm}$ at $25^{\circ} \mathrm{C}$. That means when the value of the integer part $p$ is larger than 30 , there is no one-to-one relation between the value of $p$ and the possible range of the group delay distance. We note that high-precision fringe peak detection methods $[27,28]$ can also be applied for the required peak evaluation accuracy.

For example, in the following case, we cannot determine the value of $p$. The calculated group delay distance is $6851 \mathrm{~nm}$ and we know that the average temperature lies between $15^{\circ} \mathrm{C}$ and $25^{\circ} \mathrm{C}$. Therefore, there are two possible solutions. One is that the value of $p$ is 31 , and the average temperature is about $15^{\circ} \mathrm{C}$. The other is that the value of $p$ is 32 , and the average temperature is about $25^{\circ} \mathrm{C}$.

The same calculations were performed for variations in pressure (a temperature of $20^{\circ} \mathrm{C}$, a pressure of $100.5-102 \mathrm{kPa}$, and $50 \%$ humidity) and variations in humidity (a temperature of $20^{\circ} \mathrm{C}$, a pressure of $101.325 \mathrm{kPa}$, and a humidity of $40-90 \%$ ). Figures 5 and 6 show the results. We can confirm that even 

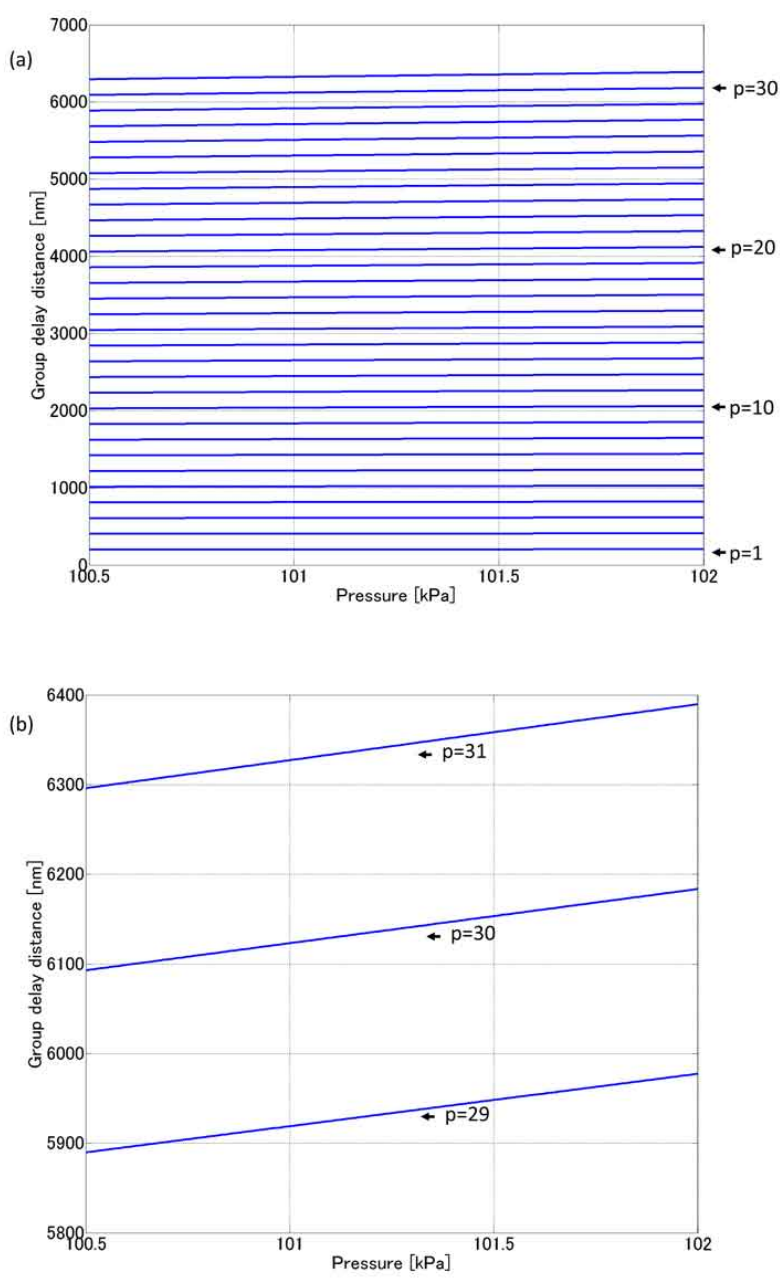

FIG. 5 (a) Calculated group delay distance versus pressure. (b) Enlarged part of (a) when the value of $p$ ranges from 29 to 31 .

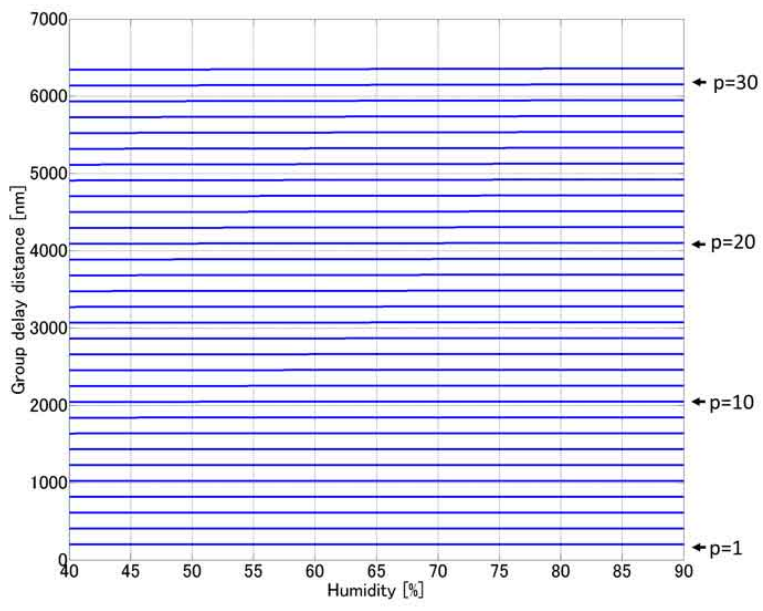

FIG. 6 Calculated group delay distance versus humidity.

when the value of $p$ is 30, the ranges of the calculated group delay distances were sufficiently separated.

Next, we calculated all combinations for the variation range of temperature $\left(15-25^{\circ} \mathrm{C}\right)$, pressure $(100.5-102 \mathrm{kPa})$, and humidity (40-90\%). The largest values of $p$ (which guaranteed that the range of group delay distance for $(p+1) \times \Lambda \times B$, $p \times \Lambda \times B$, and $(p-1) \times \Lambda \times B$ are separated) for all combinations are listed in Tables $1-3$. Because the minimum of the separable value of the integer part $p$ is 30 , the longest measurable range by the proposed method is $4.24 \times 30 \mathrm{~m} \approx 127 \mathrm{~m}$ (round trip). Note that this longest measurable range is affected by the center wavelengths, the measurement accuracy of environmental parameters, and the size of the APRIL.

\begin{tabular}{|l|c|c|c|}
\hline & & \multicolumn{2}{|c|}{ Pressure } \\
\hline & & $100.5[\mathrm{kPa}]$ & $102[\mathrm{kPa}]$ \\
\hline \multirow{2}{*}{ Humidity } & $40[\%]$ & 30 & 30 \\
& $90[\%]$ & 32 & 32 \\
\hline
\end{tabular}

TABLE 1 Largest value of $p$ under temperature variation.

\begin{tabular}{|l|c|c|c|}
\hline & & \multicolumn{2}{|c|}{ Temperature } \\
\hline & & $15\left[{ }^{\circ} \mathrm{C}\right]$ & $25\left[{ }^{\circ} \mathrm{C}\right]$ \\
\hline \multirow{2}{*}{ Humidity } & $40[\%]$ & 67 & 67 \\
& $90[\%]$ & 67 & 67 \\
\hline
\end{tabular}

TABLE 2 Largest value of $p$ under pressure variation.

\begin{tabular}{|c|c|c|c|}
\hline & & \multicolumn{2}{|c|}{ Temperature } \\
\hline & & $15\left[{ }^{\circ} \mathrm{C}\right]$ & $25\left[{ }^{\circ} \mathrm{C}\right]$ \\
\hline \multirow{2}{*}{ Pressure } & $100.5[\mathrm{kPa}]$ & 442 & 238 \\
& $102[\mathrm{kPa}]$ & 451 & 242 \\
\hline
\end{tabular}

TABLE 3 Largest value of $p$ under humidity variation.

\section{CONCLUSION}

We examined the possibility of using dispersion-induced group delay to solve the integer ambiguity problem for measuring length by an APRIL for an FOFC. Through a numerical simulation, we confirmed that there was a proportional relationship between the group delay distance and the value of the integer part. In a well-controlled laboratory or open-air conditions (i.e., a temperature of $15-25^{\circ} \mathrm{C}$, a pressure of $100.5-102 \mathrm{kPa}$, and $40-90 \%$ humidity), we can obtain the value of integer parts smaller than 30 by obtaining the group delay distance. By improving the measurement accuracy of the environmental parameters, we may expect an expansion of the measurement range. The estimation procedure of the integer part that was proposed here will aid in FOFC-based high-precision length measurements.

\section{ACKNOWLEDGEMENTS}

This research work was partially financially supported by a grant from the Support Center for Advanced Telecommunications Technology Research (SCAT)(No. H26-1).

\section{References}

[1] D. Wei, and M. Aketagawa, "Comparison of length measurements provided by a femtosecond optical frequency comb," Opt. Express 22, 7040-7045 (2014).

[2] J. Ye, "Absolute measurement of a long, arbitrary distance to less than an optical fringe," Opt. Lett. 29, 1153-1155 (2004). 
[3] D. Wei, S. Takahashi, K. Takamasu, and H. Matsumoto, "Time-offlight method using multiple pulse train interference as a time recorder," Opt. Express 19, 4881-4889 (2011).

[4] C. Narin, T. Satoru, T. Kiyoshi, and M. Hirokazu, "A new method for high-accuracy gauge block measurement using $2 \mathrm{CHz}$ repetition mode of a mode-locked fiber laser," Meas. Sci. Technol. 23, 054003 (2012).

[5] G. Wu, K. Arai, M. Takahashi, H. Inaba, and K. Minoshima, “Highaccuracy correction of air refractive index by using two-color heterodyne interferometry of optical frequency combs," Meas. Sci. Technol. 24, 015203 (2013).

[6] H. Wu, F. Zhang, S. Cao, S. Xing, and X. Qu, "Absolute distance measurement by intensity detection using a mode-locked femtosecond pulse laser," Opt. Express 22, 10380-10397 (2014).

[7] D. Wei, S. Takahashi, K. Takamasu, and H. Matsumoto, "Analysis of the temporal coherence function of a femtosecond optical frequency comb," Opt. Express 17, 7011-7018 (2009).

[8] X. Wang, S. Takahashi, K. Takamasu, and H. Matsumoto, "Spatial positioning measurements up to $150 \mathrm{~m}$ using temporal coherence of optical frequency comb," Precis. Eng. 37, 635-639 (2013).

[9] W. Sudatham, H. Matsumoto, S. Takahashi, and K. Takamasu, "Verification of the positioning accuracy of industrial coordinate measuring machine using optical-comb pulsed interferometer with a rough metal ball target," Precis. Eng. 41, 63-67 (2015).

[10] J. Zhu, P. Cui, Y. Guo, L. Yang, and J. Lin, "Pulse-to-pulse alignment based on interference fringes and the second-order temporal coherence function of optical frequency combs for distance measurement," Opt. Express 23, 13069-13081 (2015).

[11] D. Wei, K. Takamasu, and H. Matsumoto, "Synthetic adjacent pulse repetition interval length method to solve integer ambiguity problem: theoretical analysis," J. Eur. Opt. Soc.-Rapid 8, 13016 (2013).

[12] X. Wang, S. Takahashi, K. Takamasu, and H. Matsumoto, "Space position measurement using long-path heterodyne interferometer with optical frequency comb," Opt. Express 20, 2725-2732 (2012).

[13] K.-N. Jo0, Y. Kim, and S.-W. Kim, "Distance measurements by combined method based on a femtosecond pulse laser," Opt. Express 16, 19799-19806 (2008).

[14] B. Petr, M. Pavel, K. Petr, and D. Miroslav, "Length and refractive index measurement by Fourier transform interferometry and frequency comb spectroscopy," Meas. Sci. Technol. 23, 094001 (2012).

[15] P. L. Bender, and J. C. Owens, "Correction of Optical Distance Measurements for the Fluctuating Atmospheric Index of Refraction," J. Geophys. Res. 70, 2461-2462 (1965).
[16] G. Wu, M. Takahashi, K. Arai, H. Inaba, and K. Minoshima, "Extremely high-accuracy correction of air refractive index using twocolour optical frequency combs," Sci. Rep. 3, (2013).

[17] D. Wei, and M. Aketagawa, "Comparison of two-color methods based on wavelength and adjacent pulse repetition interval length," J. Eur. Opt. Soc.-Rapid 9, 14031 (2014).

[18] D. Wei, M. Aketagawa, K. Takamasu, and H. Matsumoto, "Twocolor absolute length measuring method based on pulse repetition interval lengths," Opt. Eng. 53, 122413 (2014).

[19] W. H. Knox, N. M. Pearson, K. D. Li, and C. A. Hirlimann, "Interferometric Measurements of Femtosecond Group Delay in OpticalComponents," Opt. Lett. 13, 574-576 (1988).

[20] S. Diddams, and J.-C. Diels, "Dispersion measurements with whitelight interferometry," J. Opt. Soc. Am. B 13, 1120-1129 (1996).

[21] D. Wei, K. Takamasu, and H. Matsumoto, "A study of the possibility of using an adjacent pulse repetition interval length as a scale using a HeliumâĂŞNeon interferometer," Precis. Eng. 37, 694-698 (2013).

[22] J. Ye, and S. T. Cundiff, Femtosecond optical frequency comb : principle, operation, and applications (Springer, New York, 2005).

[23] B. E. A. Saleh, and M. C. Teich, Fundamentals of photonics, Wiley series in pure and applied optics (Wiley-Interscience, Hoboken, 2007).

[24] J. A. Stone, and J. H. Zimmerman, "Refractive index of air calculator," http://emtoolbox.nist.gov/Wavelength/Edlen.asp.

[25] D. Wei, and M. Aketagawa, "Uncertainty in length conversion due to change of sensitivity coefficients of refractive index," opt. Commun. 345, 67-70 (2015).

[26] P. Balling, P. Kren, P. Masika, and S. A. van den Berg, "Femtosecond frequency comb based distance measurement in air," Opt. Express 17, 9300-9313 (2009).

[27] K. G. Larkin, "Efficient nonlinear algorithm for envelope detection in white light interferometry," J. Opt. Soc. Am. A 13, 832-843 (1996).

[28] M. C. Park, and S. W. Kim, "Direct quadratic polynomial fitting for fringe peak detection of white light scanning interferograms," Opt. Eng. 39, 952-959 (2000). 